\title{
AUTOMAÇÃO DO DESIGN POR PROGRAMAÇÃO NA MODELAGEM 3D: UMA BREVE REVISÃO
}

Thomaz Barros de Carvalho Prado ${ }^{1}$; Lilian Lefol Nani Guarieiro²

'SENAI-CIMATEC. Avenida Orlando Gomes, 1845. Piatã; Salvador/Bahia; thomaprado@gmail.com

${ }^{2}$ SENAI-CIMATEC. Avenida Orlando Gomes, 1845. Piatã; Salvador/Bahia

Resumo: Este artigo trata de uma breve revisão sistemática sobre o uso da programação de computadores para automação de modelagem 3D na indústria automotiva. Este estudo contribui para o entendimento das técnicas de programação que estão sendo usadas bem como o ambiente de desenvolvimento de produto que elas estão inseridas e como a modelagem 3D está sendo beneficiada.

Palavras-Chave: Script;Automotive;KBE;Knowledge-Based Engineering;Catia

\section{DESIGN AUTOMATION BY PROGRAMMING BASED ON 3D MODELING: A SHORT SYSTEMATIC REVIEW}

\begin{abstract}
This article presents a short systematic revision regarding the usage of computer programming aim the 3D modeling automation focused at automotive industry. This study add to the understanding of how the computer programming technics are being used, how it is inserted within the product development context and how it can favor the 3D modeling.
\end{abstract}

Keywords: Script;Automotive;KBE;Knowledge-Based Engineering;Catia 


\section{INTRODUÇÃO}

Devido a ascensão de novas tecnologias, um novo jeito de pensar e uma maior globalização dos parques indústrias, um mercado consumidor mais dinâmico e exigente, o desenvolvimento e fabricação de automóveis alcançou um novo patamar de complexidade, onde decisões precisam ser tomadas rapidamente para o rápido desenvolvimento do novo produto (do conceito até sua entrada no mercado). Dinamismo e flexibilidade são necessários a fim de mitigar a volatilidade do mercado consumidor que vem passando por mudanças cada vez mais dramáticas de seu mindset e desejo de consumo.

No processo de gestão de desenvolvimento de produto existe, dentre outras etapas, a fase virtual de desenvolvimento do novo produto automotivo (Figura 1). É nesta fase que se concentram os esforços para uma pré-visualização dos resultados, de modo a conter quaisquer imprevistos na fabricação final do produto e o fechamento da equação financeira do produto, ocorrendo dessa forma a validação virtual do produto [1].

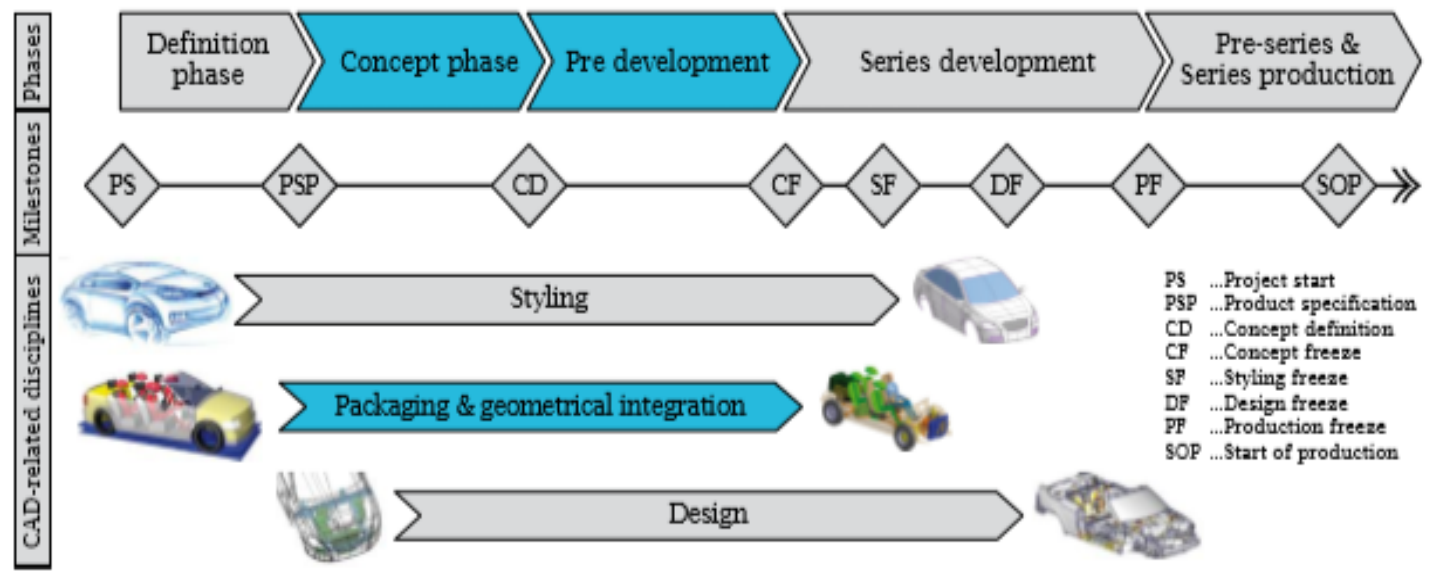

Figura 1. Processo de desenvolvimento automotivo [1].

A validação virtual do produto vem se tornando uma das fases mais crítica de todo um projeto veicular, nela a engenharia tem a flexibilidade de fazer, testar e refazer quaisquer elementos automotivos que por ventura venha a apresentar resultados que não atendam aos requisitos previamente definidos no pré-projeto, bem como a absorção de quaisquer alterações de escopo de projeto que venha a surgir.

$\mathrm{O}$ desenvolvimento de produto convencional se baseia em design-build-test, onde a performance e a confiabilidade do produto dependem pesadamente de testes físicos, o que envolve fabricação de protótipos, os quais são considerados caros e demorados, especialmente quando há a necessidade de alterações de design para correção de problemas físicos [2]. Segundo CHANG [2] o sistema do processo convencional de desenvolvimento, usualmente conduzido de forma sequencial, sofre com o design paradox, o qual se refere a dicotomia entre o conhecimento do designer sobre o produto/peça e o número de decisões realizadas durante o ciclo de desenvolvimento de produto (Figura 2a). Um dos problemas enfrentados no design paradox (Figura 2a) é que a maioria das decisões de design são tomadas em estágios 
iniciais do desenvolvimento do produto, ou seja, muito do design do produto e suas interfaces ainda não são conhecidas, e consequentemente alterações são requisitadas com frequência e de forma tardia. Dessa forma, aumentando os custos do projeto dentro do seu ciclo de desenvolvimento (Figura 2b).

Figura 2. (a) Paradoxo do Design [2]; (b) Custos/ECR versus Tempo de projeto em
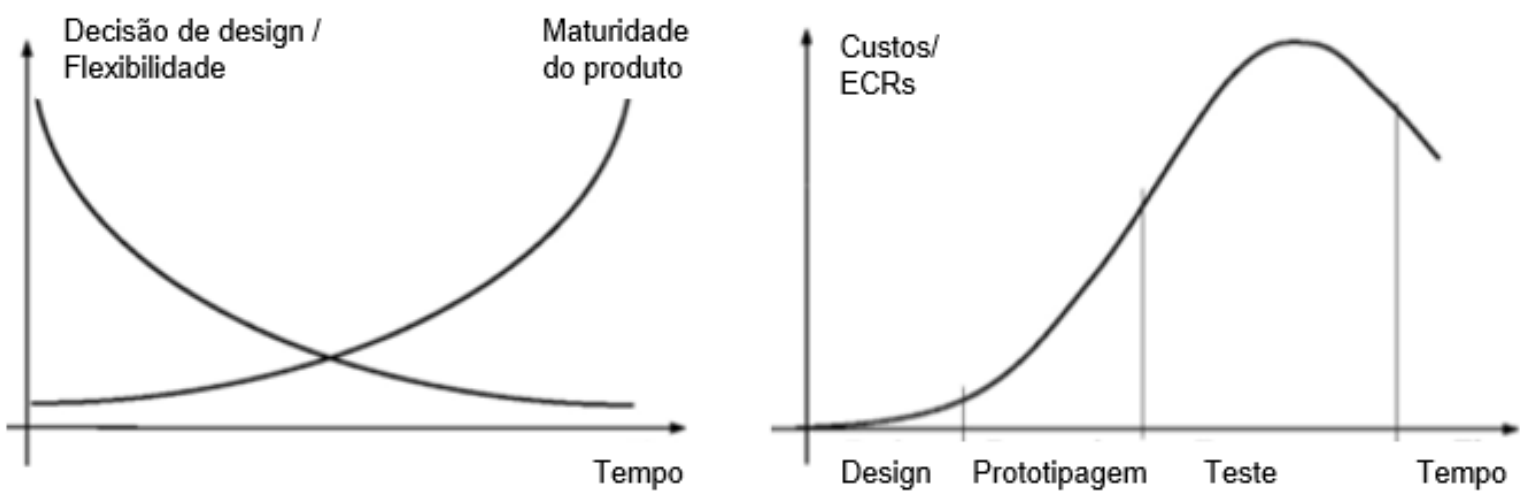

um ciclo convencional

(a)

(b)

Observando a Figura 2a, nota se que os custos das alterações de engenharia (ECRs) no design durante a fase de prototipagem e teste sobem de forma considerável. Assim, tornando o desenvolvimento do produto altamente custoso e demorado para a realidade atual. Neste contexto, novas estratégias precisam ser utilizadas para a entrega de um produto melhor, de forma mais rápida e de melhor qualidade. Dentre as novas estratégias pode-se destacar o uso da prototipagem virtual, onde pelo uso de ferramentas apoiadas, utilizando um computador, há uma melhor gestão de recursos e uma diminuição da prototipagem físicas do produto. Durante a validação virtual de produto, softwares são utilizados de forma combinada para execução de malhas de computer-aided engineering (CAE) e computer-aided manufacturing (CAM), integração antes somente possível por meio da construção física de protótipos [2]. Na Figura 3a é possível visualizar tal correlação.

Figura 3. (a) Custos/ECR versus Tempo no e-Design; (b) Product Knowledge versus Tempo no e-Design. 


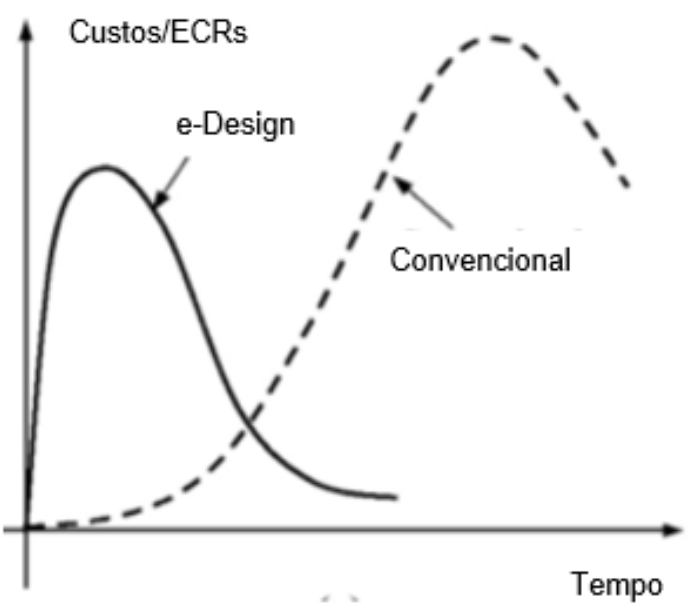

(a)

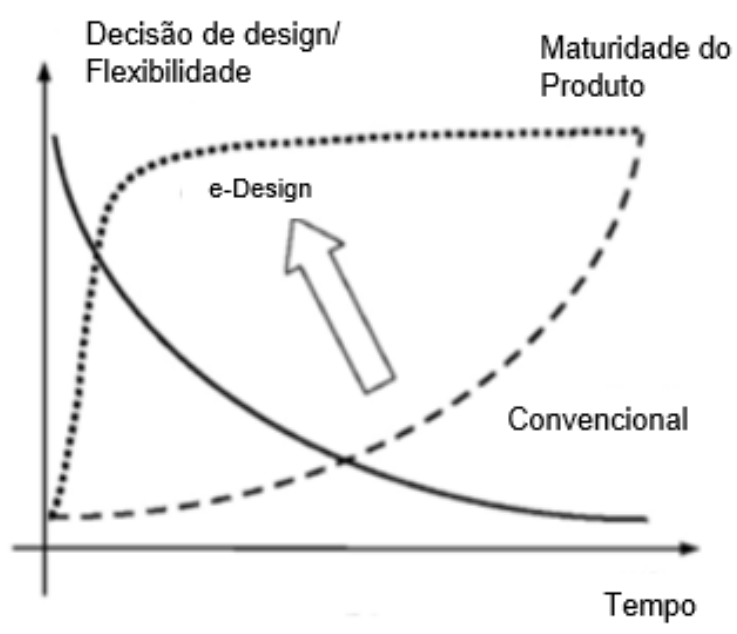

(b)

A integração das diversas tecnologias de software e seu uso no desenvolvimento de produto mais enxuto foi nomeado por CHANG [2] como e-Design. Contudo, para FILHO [3], CHAPMAN e PINFOLD[4] esse conceito é melhor definido como Knowledge Based Engineering (KBE), que representa o próximo passo da evolução do sistemas CAx Systems [3], sendo a união entre a programação orientada a objeto (POO), inteligência artificial (IA) e tecnologias de computer-aided design (CAD), Figura 3b.

Segundo CHAPMAN e PINFOLD [4], um sistema KBE objetiva a compilação das informações do produto e processo de forma que se permita modelar a engenharia envolta no processo de desenvolvimento do produto. Assim, é possível usar este conhecimento compilado para a criação de modelos que automatizem o design ou partes dele. Ainda segundo CHAPMAN e PINFOLD [4], o objetivo final do KBE é capturar as melhores práticas de design expertise de engenharia compilando-as a uma base de com conhecimento (knowledge base). Dessa forma, provendo um framework formal de captura e definição de processo para criação de design. Assim, reduzindo o time-to-product time frame, encurtando o tempo de desenvolvimento do CAD e praticamente eliminando tarefas repetitivas, o que possibilita o uso da equipe de design em outras funções, propiciando mais tempo no processo criativo e consequentemente um ambiente de inovação [3].

Neste contexto, o objetivo deste trabalho foi realizar uma breve revisão sistemática da literatura visando a prospecção de aplicações e pesquisadores que contribuem para o desenvolvimento de pesquisas relacionadas a este tema.

\section{METODOLOGIA}

O processo de revisão sistemática foi desenvolvido utilizando as bases de dados ScienceDirect (www.sciencedirect.com), SAE (www.sae.com) e Cad Journal (http://www.cad-journal.net/). Os descritores de buscas utilizados foram: Script; 
Automotive; KBE; Knowledge-Based Engineering; Catia. Os operadores booleanos AND e OR foram utilizados para se obter o maior número de resultados possíveis sobre o tema da pesquisa.

No processo de revisão sistemática foram definidos critérios de inclusão e exclusão das publicações. Assim, fizeram parte do escopo de artigos para 0 desenvolvimento do estudo aqueles que fossem: publicados nos últimos 10 anos, artigos com referência ao software CATIAV5 por ser a ferramenta de modelagem mais utilizada pelos principais OEMs automotivos [3] e publicações nos idiomas português e inglês. As publicações que não se enquadraram nos filtros descritos anteriormente foram desconsideradas.

Foram definidas as seguintes questões para avaliação da qualidade e escopo das publicações encontradas, avaliando-se o resumo, a introdução e conclusão: (i) Houve a fundamentação sobre KBE; (ii) Houve o desenvolvimento de script; (iii) Foram apresentados os resultados do uso do script.

\section{RESULTADOS E DISCUSSÃO}

Através da revisão sistemática e utilização de filtros apropriados para o tema da presente pesquisa, foram obtidos um total de 31 artigos científicos. Em termos de número de publicações nos últimos dez anos, foi possível perceber um aumento do interesse sobre o tema nos últimos 5 anos (Figura 4).

Figura 4: Distribuição das publicações sobre o tema da pesquisa por ano.

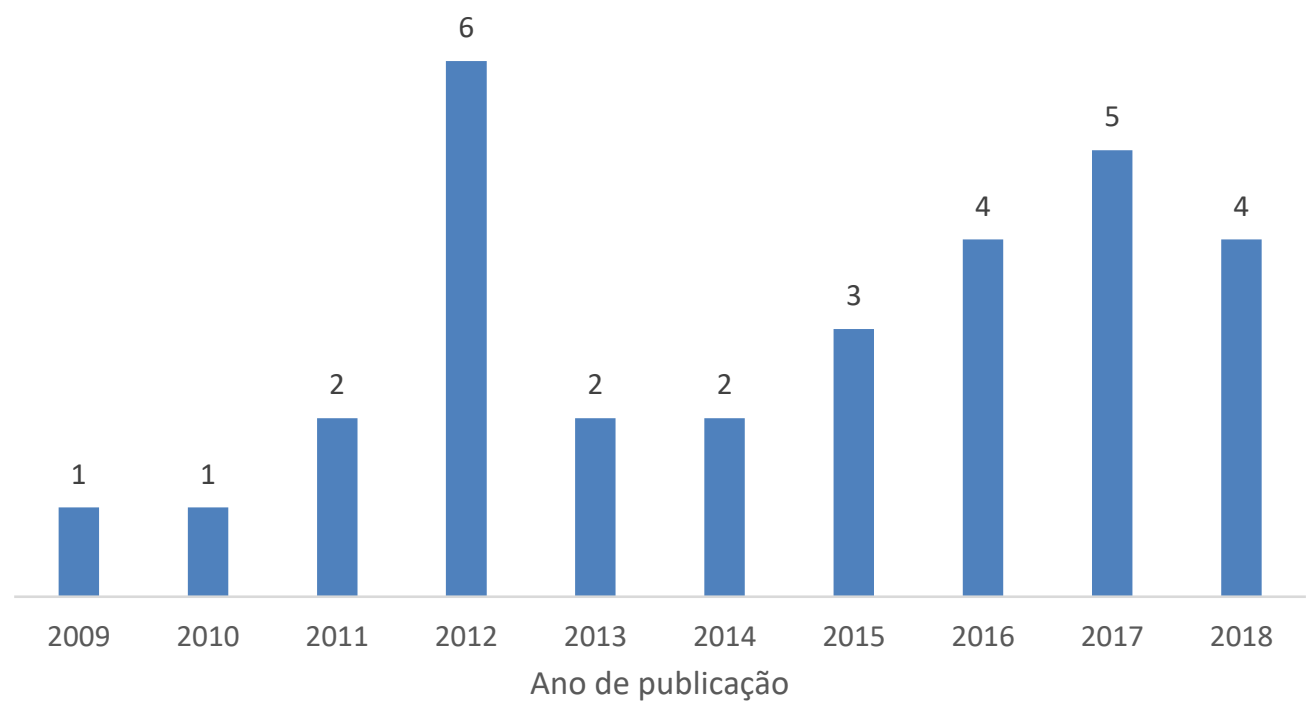

Observa-se no gráfico da Figura 4 um aumento no número de publicações relacionadas ao uso da metodologia KBE no desenvolvimento de modelos 3D e em validações em ambientes 3D. É importante o observar que o ano de 2019 não retornou resultados, uma das hipóteses é que os artigos para o ano em questão ainda não foram disponibilizados nas bases de dados. 
Dentre os artigos selecionados nota-se uma relevante contribuição de autores de países europeus, em especial a Áustria por meio da Universidade de Tecnologia de Graz tendo como seu principal contribuidor o pesquisador Mario Hirz, Ph.D.; sendo como autor principal ou coautor.

Este resultado é interessante pois a Áustria não é um país de tradição automotiva e não possui em seu território grandes montadoras ou centros de desenvolvimento instalados, logo este resultado é algo que deve ser estudado melhor em publicações futuras. A despeito das observações realizadas até o momento, é importante ressaltar que independentemente da aplicação a junção da programação com a engenharia de produto é responsável pelas bases para a criação de um KBE, que por sua vez vem sendo cada vez mais utilizado dentro da indústria como meio de automatizar rotinas repetitivas e manuais que são realizadas pelos designers de produto. Com a automação dessas rotinas tem-se um ganho de tempo e confiabilidade do modelo 3D final uma vez que as técnicas de modelagem previamente incorporadas na modulo KBE são técnicas interdisciplinares e já validadas pela engenharia.

Dessa forma, o design final de um modelo sempre estará alinhado com a últimas práticas de engenharia e não mais preso ao conhecimento de indivíduos específicos na organização, dessa forma o KBE ainda atua como meio de multiplicação do conhecimento dentro de um ambiente de desenvolvimento do produto.

Como exemplos da aplicação do KBE destacamos em ERNST et al [5] a aplicação no estudo virtual para o teste de ocorrência de danos a pintura veicular via o lançamento de pedras pelo pneu dianteiro do automóvel.

Já em MENANTEAU [6], ocorre a aplicação de técnicas para o desenvolvimento de um módulo KBE que é responsável por modelar o correto dimensionamento de um tubo de freios. Dentro os 31 artigos selecionados e nos demais que ficaram fora da seleção, nota-se a aplicação do conceito de KBE nos mais diversos nichos, e ainda com um grande potencial a ser explorado, já que como visto anteriormente a fase virtual tende a se sobrepor a fase de prototipagem física dos produtos, sejam eles automotivos ou não, sendo a metodologia KBE um vetor de otimização para a construção de modelos 3D.

\section{CONCLUSÃO}

Observou-se com este estudo que com o uso de técnicas de programação aliadas ao conhecimento prévio de engenharia é possível desenvolver métodos automatizados para a confecção de modelos 3D de forma mais rápida e com maior nível de confiabilidade.

Verificou-se ainda que o uso do KBE está avançando na para o design de modelos mais complexos, se hoje o KBE é um suporte ao desenvolvimento de modelos 3D, notou-se durante a leitura das publicações selecionadas que o KBE tem o potencial para ser utilizado no desenvolvimento de modelos 3D em sua totalidade, saindo de um cenário de suporte para um ambiente em que o KBE é o protagonista. 


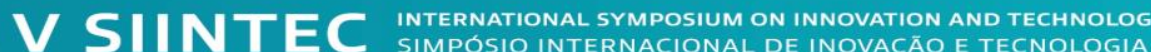

\begin{tabular}{l|l} 
CIRCULAR ECONOMY & ECONOMIA CIRCULAR
\end{tabular}

Uma vez deixando de ser uma metodologia de suporte ao desenvolvimento de produtos automotivos o KBE se alinha perfeitamente no conceito de automação do design e por sua vez na filosofia que hoje traduz a indústria 4.0, uma vez que há um ganho e uma oportunidade substancial de se aplicar as técnicas de inteligência artificial, machine learning, entre outros. Permitindo, por fim, não só a automação do design, mas sim, a automação inteligente do design permitindo a evolução dos módulos KBE a partir não só do input externo, via a criação de novas funções e linhas de código, mas também contando com técnicas que permitam o código evoluir conforme aplicado.

\section{REFERÊNCIAS}

[1] HIRZ, Mario, et al. Conceptual Full-Vehicle Development supported by Integrated Computer-Aided Design Methods. Computer-Aided Design and Applications. 10. 159-172. 2013.

[2] CHANG, Kuang-Hua. Product Manufacturing and Cost Estimating using CAD/CAE. Chapter 1 - Introduction to e-Design. Elsevier. Oxford, 2013.

[3] FILHO, Rogerio Percio Bahr. WELLER, Thiago Rodrigues. Automotive Industry CAD System Automation through Knowledge Based Engineering (KBE) case study. SAE Technical Paper Series. 2016.

[4] CHAPMAN, C.B, PINFOLD, M. Design engineering-a need to rethink the solution using knowledge based engineering. Knowledge-Based Systems 12. 257-267. 1999

[5] ERNST, Markus et al. A Method of CAD Based Automation and Simulation by the Example of Virtual Stone Chipping Testing. Computer-Aided Design \& Applications 11. 295-304. 2013.

[6] MENANTEAU, Laurent. COLSOUL, Frederic. Pipes Modeling in the Automotive Field. SAE International. 2012-01-0451. 2012

[8] RUSCHITZKA, Margot et al. Ontologically-based approach for Knowledge Representation for Stamping Die Applications. Computer-Aided Design \& Applications 6. 281-289. 2009.

[9] STADLER, Severin. HIRZ, Mario. A knowledge-based framework for integration of computer aided styling and computer aided engineering. Computer-Aided Design \& Applications 13. 558-569. 2016.

[10] SALCHNER, Markus et al. A new approach of a global knowledge-based engineering infrastructure. Computer-Aided Design \& Applications 14. 366-376. 2017.

[11] HAGENREINER, Thomas and KÖHLER, Peter. Concept Development of Design Driven Parts Regarding Multidisciplinary Design Optimization. Computer-Aided Design \& Applications 12. 208-217. 2014. 
[12] KREIS, Alexander et al. Convenient connection technology data model supporting optimized information exchang between CAx-systems. Computer-Aided Design \& Applications 15. 771-778. 2018.

[13] BODEIN, Yannick et al. Decisional Model for KBE Implementation in a Commercial CAD Software. Computer-Aided Design \& Applications 9. 121-131. 2012.

[14] RENZI, Cristina et al. Integrated geometrical and dimensional tolerances stack-up analysis for the design of mechanical assemblies: an application on marine engineering. Computer-Aided Design \& Applications 15. 631-642. 2018.

[15] TSAI, Yi-Lung et al. Knowledge-based Engineering for Process Planning and Die Design for Automotive Panels. Computer-Aided Design \& Applications 7. 75-87. 2010.

[16] SALCHNER, Markus et al. Multi-CAD approach for knowledge-based design method. Computer-Aided Design \& Applications 13. 471-483. 2016.

[17] MAS, F. et al. A review of PLM impact on US and EU Aerospace Industry. Procedia Engineering 132. 1053-1060. 2015.

[18] DEMOLY, Frédéric et al. An assembly oriented design framework for product structure engineering and assembly sequence planning. Robotics and ComputerIntegrated Manufacturing 27. 33-46. 2011.

[19] JOHANSSON, Christian et al. Product-Service Systems for Functional Offering of Automotive Fixtures: Using Design Automation as Enabler. Procedia CIRP 64. 411416. 2017.

[20] BODEIN, Yannick Bodein et al. A roadmap for parametric CAD efficiency in the automotive industry. Computer-Aided Design 10. 1198-1214. 2013

[21] ROCCA, Gianfranco La. Knowledge based engineering: Between Al and CAD. Review of a language based technology to support engineering design. Advanced Engineering Informatics 26. 159-179. 2012.

[22] OLOFSSON, Jakob et al. A methodology for microstructure-based structural optimization of cast and injection molded parts using knowledge-based design automation. Advances in Engineering Software 109. 44-52. 2017.

[23] FERREIRA, Filipe et al. Product lifecycle management in knowledge intensive collaborative environments: An application to automotive industry. International Journal of Information Management 37. 1474-1487. 2017.

[24] AMATE-QUINTANA, S. et al. Transforming expertise into Knowledge-Based Engineering tools: A survey of knowledge sourcing in the context of engineering design. Knowledge-Based Systems 84. 89-97. 2015.

[25] LOIS-SIDNEY, Ludovic Louis-Sidney et al. A conceptual model for the implementation of an Inter-Knowledge Objects Exchange System (IKOES) in automotive industry. Engineering Applications of Artificial Intelligence 25. 1090-1101. 2012. 